\title{
On formal Riemannian metrics
}

\author{
Mihaela Pilca
}

\begin{abstract}
Formal Riemannian metrics are characterized by the property that all products of harmonic forms are again harmonic. They have been studied over the last ten years and there are still many interesting open conjectures related to geometric formality.

The existence of a formal metric implies Sullivan's formality of the manifold, and hence formal metrics can exist only in presence of a very restricted topology.

In this paper we give an overview over the present state of research on geometrically formal manifolds, with emphasis on the recent results obtained by the author together with Liviu Ornea in [11]. We are mainly interested in the topological obstructions to the existence of formal metrics. Moreover, we discuss natural constructions of formal metrics starting from known ones.
\end{abstract}

\section{Motivation, Definitions and Examples}

A manifold is geometrically formal if it admits a formal Riemannian metric, while a Riemannian metric is called formal if all products of harmonic forms are again harmonic. One of the main motivation for the study of such manifolds is that the existence of a formal metric implies Sullivan's formality of the manifold.

In algebraic topology one wants to read the homotopy type of a space in terms of cohomological data. A precise definition of this property was given

Key Words: geometric formality, topological formality, Betti numbers, harmonic form, warped product, conformal metrics, Vaisman manifold.

2010 Mathematics Subject Classification: Primary 53C25; Secondary 53C55, 58A14.

Received: August, 2011.

Accepted: February, 2012. 
by Sullivan in [13] and is called formality. Roughly, a formal manifold is one whose real (or rational) homotopy type is a formal consequence of its real (resp. rational) cohomology ring. Algebro-topologically this means that all Massey products vanish. The rational homotopy theory is the study of the rational homotopy type of a space, which mainly means that all torsion in the homotopy groups is ignored. The principal idea is that rational homotopy types of simply connected spaces can be identified with certain algebraic objects called minimal Sullivan algebras, which are commutative differential graded algebras over the rational numbers satisfying certain conditions.

As far as manifolds are concerned, it is known e.g. that all compact Riemannian symmetric spaces and all compact Kähler manifolds are formal.

Sullivan also observed that if a compact manifold admits a metric such that the wedge product of any two harmonic forms is again harmonic, then, by Hodge theory, the manifold is formal. This motivated Kotschick to give the following:

Definition $1.1([6])$. A Riemannian metric is called formal if all wedge products of harmonic forms are harmonic. A closed manifold is called geometrically formal if it admits a formal Riemannian metric.

Let us first fix the notation. We denote by $\left(M^{n}, g\right)$ a compact oriented Riemannian $n$-dimensional manifold without boundary and by $\Omega^{p}(M), 0 \leq$ $p \leq n$ the space of smooth, real valued, $p$-forms of $M$. The de Rham complex

$$
\ldots \rightarrow \Omega^{p}(M) \stackrel{d}{\rightarrow} \Omega^{p+1}(M) \rightarrow \ldots
$$

where $d$ stands for exterior derivative is then used to introduce the de Rham cohomology groups $H_{d R}^{p}(M)$. The topological information contained in these cohomology groups may be understood geometrically, using Hodge theory, by means of the isomorphisms

$$
H_{d R}^{p}(M) \cong \mathcal{H}^{p}(M, g), 0 \leq p \leq n,
$$

where the space of harmonic $p$-forms of $\left(M^{n}, g\right)$ is defined by

$$
\mathcal{H}^{p}(M, g)=\left\{\alpha \in \Omega^{p}(M) \mid \Delta \alpha=0\right\},
$$

for the Laplacian on forms: $\Delta=d \delta+\delta d$, where $\delta$ is the formal adjoint of $d$ with respect to the given metric and orientation. We now set

$$
H_{d R}^{\star}(M)=\bigoplus_{p \geq 0} H_{d R}^{p}(M) \text { and } \mathcal{H}^{\star}(M, g)=\bigoplus_{p \geq 0} \mathcal{H}^{p}(M, g) .
$$

Whereas $H^{\star}(M)$ is a graded algebra, in general $\mathcal{H}^{\star}(M, g)$ is not an algebra with respect to the wedge product operation for there is no reason that the isomorphism (1.1) descends to the level of harmonic forms. 
Thus, in general, on a Riemannian manifold, wedge products of harmonic forms are not usually harmonic, so $\mathcal{H}^{*}(M, g)$ is not closed under wedge product. But there are some examples where this does happen, for instance arbitrary Riemannian metrics on rational homology spheres. Classical examples of geometrically formal manifolds are compact globally symmetric spaces. On these manifolds the harmonic forms coincide with the invariant ones, and the latter are clearly closed under products.

In [7] and [8] more general examples are provided, both of geometrically formal and of formal but non-geometrically formal homogeneous manifolds.

An important property of formal metrics is that the length of any harmonic form is (pointwise) constant. This is a striaghtforward consequence of the definition and the formula $\alpha \wedge * \alpha=|\alpha|^{2} \operatorname{vol}_{g}$ (where $\operatorname{vol}_{g}$ is the volume form which is harmonic) applied to any harmonic form $\alpha$ (then $* \alpha$ is also harmonic since $\Delta$ commutes with the Hodge-* operator). Furthermore, by polarisation, it follows that the inner product of any two harmonic forms is constant. This has important consequences in many arguments, for instance the linear independence of a set of harmonic forms is sufficient to be tested at one point.

This basic property also places the formal metrics into the larger class of metrics having all harmonic forms of constant length. These naturally appear in other geometric contexts, for instance in the study of certain systolic inequalities, and has been investigated in [9], [10]. Following [9] we now introduce various notions of "constancy" related to harmonic forms.

Definition 1.2. Let $\left(M^{n}, g\right)$ be compact and oriented. It is said to satisfy the hypothesis $\left(C L_{p}\right)$ for some $1 \leq p \leq n-1$ if and only if every harmonic $p$-form has pointwisely constant norm.

Manifolds satisfying hypothesis $\left(C L_{1}\right)$ appear to be naturally related to a generalized systolic inequality. More precisely, for a compact, orientable Riemannian manifold $\left(N^{n}, g\right)$ with non-vanishing first Betti number one defines the stable 1-systole $\operatorname{stsys}_{1}(\mathrm{~g})$ in terms of the stable norm. If $\operatorname{sys}_{n-1}(\mathrm{~g})$ is the infimum of the $(n-1)$-volumes of all nonseparating hypersurfaces in $N$, then the following systolic inequality holds:

$$
\operatorname{stsys}_{1}(g) \cdot \operatorname{sys}_{n-1}(g) \leq \gamma_{b_{1}(N)}^{\prime} \cdot \operatorname{vol}(g) .
$$

Here $\gamma_{b_{1}(N)}^{\prime}$ is the Bergé-Martinet constant for whose definition we refer to [2]. The important point for us is that it was shown in [2] that if equality in (1.2) occurs then $\left(N^{n}, g\right)$ satisfies the hypothesis $\left(C L_{1}\right)$. Note that the converse is false, as flat tori always satisfy $\left(C L_{1}\right)$ but saturate $(1.2)$ if and only if they are dual-critical. 
Riemannian manifolds $\left(N^{n}, g\right)$ satisfying equality in (1.2) have strong geometric properties. It was proved in [2], Thm. 1.2, that in this case $\left(N^{n}, g\right)$ is the total space of a Riemannian submersion with minimal fibers to a flat torus, whose projection is actually the Albanese map. Therefore, in the special case when $b_{1}(N)=n-1$ it follows that the fibers of the Albanese map must be totally geodesic. Using Chern-Weil theory and an argument that reproduces in part that in section 6 of [2], it was shown in [10] that the only possible topologies of manifolds $N^{n}$ which admit a metric satisfying $\left(C L_{1}\right)$ and have $b_{1}(N)=n-1$ are those of 2-step nilmanifolds with 1-dimensional kernel. Equivalently, the above class of manifolds is parametrized by couples $(T, \omega)$ where $T$ is a flat $(n-1)$-torus and $\omega$ is a non zero, integral cohomology class on $T$. The next definition, which is a kind of refinement of Definition 1.1, is related to this fact.

Definition 1.3. Let $\left(M^{n}, g\right)$ be compact and oriented. The metric $g$ is $p$ formal for some $1 \leq p \leq n-1$ if and only if the product of any harmonic p-forms remains harmonic.

As noticed above, formal metrics satisfy hypothesis $\left(C L_{p}\right)$ for all $1 \leq p \leq$ $n-1$. However, the converse is not true: the class of (non necessarily invariant) metrics on nilmanifolds studied in [10] satisfy hypothesis $\left(C L_{p}\right)$ whenever $1 \leq p \leq n-1$, but none of the $p$-formality hypothesis. Moreover, it is known that certain classes of homogeneous spaces fail to be geometrically formal for cohomological reasons [7].

One of the natural questions which arises is which constructions allow to obtain new examples from known ones. We considered the following elementary constructions:

(i) Riemannian Products. By considering the splitting of the codifferential on a product of Riemannian manifolds and the corresponding commutation relations with the natural projections on the factors, a direct computation yields the following:

Proposition 1.4. If $\left(M_{1}, g_{1}\right)$ and $\left(M_{2}, g_{2}\right)$ are two compact Riemannian manifolds with formal metrics, then the metric $g=g_{1}+g_{2}$ on the product manifold $M=M_{1} \times M_{2}$ is also formal.

(ii) Warped Products. In this case, also by a direct but rather lenghty computation, we have shown the following (for the proof we refer to [11]):

Theorem $1.5([11])$. Let $\left(B^{n}, g_{B}\right)$ and $\left(F^{m}, g_{F}\right)$ be two compact Riemannian manifolds with formal metrics. Then the warped product metric $g=\pi^{*}\left(g_{B}\right)+$ $(\varphi \circ \pi)^{2} \sigma^{*}\left(g_{F}\right)$ on $B \times{ }_{\varphi} F$ is formal if and only if the warping function $\varphi$ is constant. 
(iii) Conformal Changes. We have studied under which condition two formal metrics may belong to the same conformal class. Under a weak topological assumption, we obtained the following uniqueness result:

Proposition 1.6 ([11]). Let $M^{2 n}$ be an even-dimensional compact manifold whose middle Betti number $b_{n}(M)$ is non-zero. Then, in any conformal class of metrics there is at most one formal metric (up to homothety).

Proof: Let $[g]$ be a class of conformal metrics on $M$ and suppose there are two formal metrics $g_{1}$ and $g_{2}=e^{2 f} g_{1}$ in $[g]$. In the middle dimension the kernel of the codifferential is invariant at conformal changes of the metric, so that there are the same harmonic forms for all metrics in a conformal class: $\mathcal{H}^{n}\left(M, g_{1}\right)=\mathcal{H}^{n}\left(M, g_{2}\right)$. As $b_{n}(M) \geq 1$ there exists a non-trivial $g_{1}$-harmonic (and thus also $g_{2}$-harmonic) $n$-form $\alpha$ on $M$. The length of $\alpha$ must then be constant with respect to both metrics, which are assumed to be formal and thus we get:

$$
g_{2}(\alpha, \alpha)=e^{2 n f} g_{1}(\alpha, \alpha),
$$

which shows that $f$ must be constant.

Remark 1.7. Using the product construction to assure that the middle Betti number is non-zero, one can build such examples of formal metrics which are unique in their conformal class. Other examples are provided by manifolds with "big" first Betti number, as follows from Corollary 2.3: if $b_{1}\left(M^{2 n}\right) \geq n$, then $b_{n}\left(M^{2 n}\right) \geq 1$.

Remark 1.8. We notice that all the above results related to constructions of new formal metrics hold true more generally for metrics having all harmonic forms of constant length.

\section{Topological Obstructions}

Geometric formality imposes strong restrictions on the (real) cohomology of the manifold. This fact was first observed by Sullivan in [13]: "There are topological obstructions for $M$ to admit a metric for which the product of harmonic forms is harmonic." For example, it is proven in [6] that a manifold admits a non-formal metric if and only if it is not a rational homology sphere.

In this article, we shall present the already known obstructions to formality and the new ones obtained in [11].

The topological restrictions satisfied by geometrically formal manifolds are similar to properties of flat manifolds, as it was noticed in [6]. It was also Kotschick who remarked that metric formality is a weakening of a reduction of holonomy. For example, as noticed above, it implies that harmonic forms have 
constant length, though it does not imply that they are parallel. Nevertheless, the more harmonic forms there are, the stronger the constraints.

Most of these obstructions are independent of formality in the sense of rational homotopy theory and are often nonzero on formal manifolds.

We first consider the existence of upper bounds for the Betti numbers and the description of the limiting manifolds. Then we look at certain other relations between the Betti numbers. More precisely, we show that on a compact geometrically formal manifold with $b_{1}=p \geq 1$, if there exist two vanishing Betti numbers such that the distance between them is not larger than $p+2$, then all the intermediary Betti numbers must be zero too.

One of the most important conjecture related to geometrically formal manifolds is that they have the same real cohomology algebra as the compact globally symmetric spaces. This is known to be true up to dimension 4 (this result is due to Kotschick, [6]) and is still open for higher dimensions. In $\S 2.3$ we shall give the main ideas of the proofs.

In the last part we look at special geometric structures and analyze the topological obstructions imposed by the formality of the Riemannian metric of the structure. More precisely, we first recall the known results about the Kähler and Sasaki structures and then consider the locally conformally Kähler (lcK) structures and in particular Vaisman manifolds. Unlike Kähler manifolds, which are known to be formal (cf. [3]), nothing is yet known about the Sullivan formality of locally conformally Kähler (in particular Vaisman) manifolds.

\subsection{Upper bounds for the Betti Numbers and limiting manifolds}

The Betti numbers of any geometrically formal manifold are bounded from above by the Betti numbers of the torus in that dimension. More precisely, the following holds:

Theorem 2.1 ([6]). On a closed oriented geometrically formal manifold $M^{n}$ the following inequalities hold:

1. The real Betti numbers of $M$ are bounded by $b_{k}(M) \leq b_{k}\left(T^{n}\right)=\left(\begin{array}{l}n \\ k\end{array}\right)$.

2. The first Betti number $b_{1}(M) \neq n-1$.

3. If $n=4 m$, then $b_{2 m}^{ \pm}(M) \leq b_{2 m}^{ \pm}\left(T^{n}\right)$.

The main idea of the proof is that the number of linear independent harmonic $k$-forms cannot exceed the rank of the bundle of $k$-forms (for details we refer to $[6])$.

We now consider the limiting manifolds for the above inequalities for the Betti numbers. Namely, a geometrically formal manifold with maximal first 
or second Betti number is shown to be flat. For the Betti numbers of higher order it is still a conjecture that the corresponding limiting manifolds are also flat.

The main result concerning geometrically formal metrics with maximal first Betti number is due to Kotschick:

Theorem $2.2([6])$. Suppose the closed oriented manifold $M^{n}$ is geometrically formal. If $b_{1}(M)=k$, then there is a smooth submersion $\pi: M \rightarrow T^{k}$, for which $\pi^{*}$ is an injection of cohomology algebras. In particular, if $b_{1}(M)=n$, then $M$ is diffeomorphic to $T^{n}$. In this case every formal Riemannian metric is flat.

The idea of the proof is to consider the Albanese map $\pi: M \rightarrow T^{k}$ given by integration of harmonic 1 -forms. The flatness of the metric then follows from the existence of a trivialization given by a basis of harmonic 1-forms.

A straightforward consequence of the above result is the following property of "propagation" for Betti numbers on geometrically formal manifolds:

Corollary 2.3. If the first Betti number of a closed oriented geometrically formal manifold $M$ satisfies $b_{1}(M)=p \geq 1$, then $b_{q}(M) \geq\left(\begin{array}{c}p \\ q\end{array}\right)$, for all $1 \leq$ $q \leq p$.

The characterisation of geometrically formal Riemannian manifold with maximal second Betti number was given by Grosjean and Nagy as follows:

Theorem 2.4 ([5]). Let $M^{n}$ be geometrically formal with $n \geq 3$. If $b_{2}(M)$ is maximal, $b_{2}(M)=\left(\begin{array}{l}n \\ 2\end{array}\right)$, then any formal metric on $M$ is flat.

The proof of Theorem 2.4 uses a nice trick when the dimension $n$ is odd, which reduces to the preceding case of maximal first Betti number. Namely, if $b_{p}(M)$ and $b_{q}(M)$ are maximal for $p=q \leq \operatorname{dim}(M)$, then also $b_{p+q}(M)$ must be maximal. Applying this to $b_{2}(M)$, we obtain in odd dimensions that $b_{1}(M)=b_{n-1}(M)$ is maximal. The even-dimensional case is more involved and uses the existence of a compatible almost Kähler structure on $M$ (see [5]).

\subsection{Vanishing of Intermediary Betti Numbers}

We further present obstructions to geometric formality obtained in [11]. We have shown that if a compact manifold with $b_{1}=p \geq 1$ admits a formal metric, and if there exist two vanishing Betti numbers such that the distance between them is not larger than $p+2$, then all the intermediary Betti numbers must be zero too. More precisely, we have: 
Proposition 2.5 ([11]). Let $M^{n}$ be a compact geometrically formal manifold with $b_{1}(M)=p \geq 1$. If there exist two Betti numbers that vanish: $b_{k}(M)=$ $b_{k+l}(M)=0$, for some $k$ and $l$ with $0<k+l<n$ and $0<l \leq p+1$, then all intermediary Betti numbers must vanish: $b_{i}(M)=0$, for $k \leq i \leq k+l$. In particular, if there exists $k \geq \frac{n-p-1}{2}$ such that $b_{k}(M)=0$, then $b_{i}(M)=0$ for all $k \leq i \leq n-k$.

Proof: Let $\left\{\theta_{1}, \ldots, \theta_{p}\right\}$ be an orthogonal basis of $g$-harmonic 1-forms, where $g$ is a formal metric on $M$. We first notice that here is no ambiguity in considering the orthogonality with respect to the global scalar product or to the pointwise inner product, because, when restricting ourselves to the space of harmonic forms of a formal metric, these notions coincide. Thus, if two harmonic forms $\alpha$ and $\beta$ are orthogonal with respect to the global product, we get: $0=(\alpha, \beta)=\int_{M}<\alpha, \beta>\operatorname{dvol}_{g}=<\alpha, \beta>\operatorname{vol}(M)$, showing that their pointwise inner product is the zero-function.

It is enough to show that $b_{k+1}(M)=0$ and then use induction on $i$. Let $\alpha$ be a harmonic $(k+1)$-form. By formality, $\theta_{1} \wedge \theta_{2} \wedge \cdots \wedge \theta_{l-1} \wedge \alpha$ is a harmonic $(k+l)$-form and thus must vanish, since $b_{k+l}(M)=0$. On the other hand, $\left.\theta_{j}^{\sharp}\right\lrcorner \alpha=(-1)^{k(n-k-1)} *\left(\theta_{j} \wedge * \alpha\right)$ is a harmonic $k$-form, again by formality. As $b_{k}(M)=0$, it follows that $\left.\theta_{j}^{\sharp}\right\lrcorner \alpha=0$, for $1 \leq j \leq p$. Then, using that $\left\{\theta_{1}, \ldots, \theta_{p}\right\}$ are also orthogonal, we obtain:

$$
\left.\left.\left.0=\theta_{1}^{\sharp}\right\lrcorner \cdots\right\lrcorner \theta_{l-1}^{\sharp}\right\lrcorner\left(\theta_{1} \wedge \cdots \wedge \theta_{l-1} \wedge \alpha\right)= \pm\left|\theta_{1}\right|^{2} \cdots\left|\theta_{l-1}\right|^{2} \alpha,
$$

which implies that $\alpha=0$, because each $\theta_{j}$ has non-zero constant length. This shows that $b_{k+1}(M)=0$.

\subsection{The Real Cohomology of Geometrically Formal Manifolds}

In small dimensions every geometrically formal manifold has the real cohomology algebra of a compact Riemannian symmetric space. However, in higher dimensions this is still an open conjecture and very few general facts are known about geometrically formal manifolds.

Theorem 2.6 ([6]). If $M$ is a closed oriented geometrically formal manifold of dimension smaller or equal to 4 , then $M$ has the real cohomology algebra of a compact globally symmetric space.

It is however not true that $M$ is a globally symmetric space, even up to homotopy. There are examples of this in dimensions 3 and 4. In [6] there are also provided examples of 4-manifolds which do have the real cohomology algebra of a compact symmetric space, but are not geometrically formal.

For the details of the proof we refer the reader to [6]. The argument is different in each dimension and the main ideas are as follows. 
- Dimension Two. If $\Sigma$ is a closed oriented surface of genus equal to 0 or 1 , then there are globally symmetric Riemannian metrics. If its genus is $\geq 2$, there are nontrivial harmonic 1 -forms for all metrics, but every 1-form has zeros. In this case every wedge product of 1-forms also has zeros, but for cohomological reasons cannot vanish identically in all cases. The only harmonic 2-forms are the constant multiples of the Riemannian volume form, so there cannot be any formal Riemannian metric.

- Dimension Three. If $M$ is a closed oriented geometrically formal 3manifold, then, by Theorem 2.1, $b_{1}(M) \in\{0,1,3\}$. If $b_{1}(M)=3$, then Theorem 2.2 implies that $M$ is the torus. If $b_{1}(M)=1$, then the real cohomology algebra is that of the globally symmetric space $S^{2} \times S^{1}$ and if $b_{1}(M)=0, M$ is a rational homology sphere.

- Dimension Four. If $M$ is a closed oriented geometrically formal 4manifold, then by Theorem 2.1 we have $b_{1}(M) \in\{0,1,2,4\}$. If the first Betti number is maximal, then Theorem 2.2 implies that $M$ is the 4torus. If $b_{1}(M)=2$ or $b_{1}(M)=1$ it is shown that the real cohomology ring of $M$ is the same as that of the globally symmetric space $S^{2} \times T^{2}$, respectively of $S^{3} \times S^{1}$. The remaining case, $b_{1}(M)=0$ is the most difficult. After an investigation of all possible cases, it turns out that all combinations occur for the globally symmetric spaces $S^{4}, \mathbb{C} P^{2}, \overline{\mathbb{C} P^{2}}$ and $S^{2} \times S^{2}$.

\subsection{Geometric formality and special geometric structures}

Kähler structures. The first special structures we consider are the Kähler structures. Compact Kähler manifolds are topologically formal in the sense of Sullivan, as it was proven in [3], but in general, they are not geometrically formal. Nagy gave in [9] algebraic and topological obstructions to the existence of a geometrically 2-formal Kähler metric, at the level of the second cohomology group. Most of these geometric properties are more generally consequences of the constancy of the length for low degree harmonic forms. The main results are the following (for their proofs we refer to [9]):

Theorem $2.7([9])$. Let $\left(M^{2 n}, g, J\right)$ be a compact Kähler manifold. Then every harmonic 1-form of pointwisely constant length is parallel with respect to the Levi-Civita connection of $g$. In particular, if $g$ satisfies the hypothesis $\left(C L_{1}\right)$ then $\left(M^{2 n}, g, J\right)$ is locally the Riemannian (and biholomorphic) product of a compact, simply connected Kähler manifold and of a flat torus.

In [9] it is also shown that harmonic 2-forms of a 2-formal Kähler manifold have a global spectral decomposition and constant eigenvalues. This sustains 
the remark, already noticed in [6], that geometric formality is weakening the notion of Riemannian holonomy reduction. Based upon this, the following result holds:

Theorem $2.8([9])$. Let $\left(M^{2 n}, g, J\right)$ be a compact Kähler manifold and assume that the metric $g$ is 2-formal. Then :

(i) the space $\mathcal{H}^{1,1}$ of $J$-invariant harmonic forms is spanned by almost Kähler forms compatible with the metric $g$.

(ii) the space $\mathcal{H}_{-}^{2}$ of $J$-anti-invariant harmonic two-forms consists only in parallel forms.

By contrast, recall that simply connected, irreducible compact Hermitian symmetric space have second Betti number equal to 1 . The case of geometrically formal Kähler manifolds of complex dimension 3 is now well understood. More precisely, the possible values of the second Betti number are known, as well as more precise information concerning the algebraic structure of the second cohomology group:

Theorem $2.9([9])$. Let $\left(M^{6}, g, J\right)$ be a geometrically formal Kähler manifold. If the metric $g$ is locally irreducible then $b_{1}(M)=b_{2}^{-}(M)=0$. Moreover one has $b_{2}(M) \leq 3$ and $\mathcal{H}^{1,1}$ is spanned by mutually commuting almost Kähler structures.

As noticed in [9], finding further, first order obstructions to geometric formality in the Kähler case relies on understanding of the algebraic structure of the space of harmonic $p$-forms, $p \geq 3$.

Sasakian structures. We further consider the topology of geometrically formal Sasakian metrics, which are the analogue of Kähler metrics in odd dimensions. The cohomology algebra of geometrically formal Sasakian manifolds can be completely described. More precisely, in [5], it is shown that a formal Sasakian metric can exist only on a real cohomology sphere.

Theorem $2.10([5])$. Let $\left(M^{2 n+1}, g\right)$ be a compact Sasakian manifold. If the metric $g$ is formal, then $b_{p}(M)=0$ for $1 \leq p \leq 2 n$, i.e. $M$ is a real cohomology sphere.

The proof uses the decomposition of the tangent bundle of a Sasakian manifold as a direct sum of the 1-dimensional distribution generated by the Reeb vector field and the horizontal contact distribution, which admits a Kähler structure. The corresponding decomposition of the exterior differential and of the codifferential acting on the space of primitive harmonic forms together 
with an algebraic relation involving powers of the Lefschetz operator yield the desired topological obstruction. A similar argument will be used below in the proof of Theorem 2.12 .

Locally conformally Kähler structures. We now discuss the geometric formality of a Vaisman manifold, which is a particular type of locally conformal Kähler (LCK) manifold. It is defined as a Hermitian manifold $(M, J, g)$, of real dimension $n=2 m \geq 4$, whose fundamental 2-form $\omega$ satisfies the conditions:

$$
d \omega=\theta \wedge \omega, \quad \nabla \theta=0 .
$$

Here $\theta$ is a (closed) 1-form, called the Lee form, and $\nabla$ is the Levi-Civita connection of the LCK metric $g$ (we always consider $\theta \neq 0$ to exclude the Kähler manifolds). Locally, $\theta=d f$ and the local metric $e^{-f} g$ is Kähler, hence the name LCK. When lifted to the universal cover, these local metrics glue to a global one, which is Kähler and acted on by homotheties by the deck group of the covering.

In the Vaisman case, the universal cover is a Riemannian cone. In fact, compact Vaisman manifolds are closely related to Sasakian ones, as the following structure theorem shows:

Theorem 2.11 ([12]). Compact Vaisman manifolds are mapping tori over $S^{1}$. More precisely: the universal cover $\tilde{M}$ is a metric cone $N \times \mathbb{R}^{>0}$, with $N$ compact Sasakian manifold and the deck group is isomorphic with $\mathbb{Z}$, generated by $(x, t) \mapsto(\lambda(x), t+q)$ for some $\lambda \in \operatorname{Aut}(N), q \in \mathbb{R}^{>0}$.

Every Hopf manifold (quotient of $\mathbb{C}^{\mathbb{N}} \backslash\{0\}$ by the cyclic group generated by a semi-simple operator with subunitary eigenvalues) is a Vaisman manifold, as well as all its compact complex submanifolds. The complete list of Vaisman compact surfaces is given in [1]. On the other hand, examples of LCK manifolds (satisfying only the condition $d \omega=\theta \wedge \omega$ for a closed $\theta$ ) which cannot admit any Vaisman metric are also known, e.g. one type of Inoue surfaces and the non-diagonal Hopf surface, see [1]. The non-diagonal Hopf surface is particularly relevant for our discussion because it is topologically formal.

Being parallel and Killing (see [4]), the Lee field $\theta^{\sharp}$ is real holomorphic and, together with $J \theta^{\sharp}$ generates a one-dimensional complex, totally geodesic, Riemannian foliation $\mathcal{F}$. Note that $\mathcal{F}$ is transversally Kähler, meaning that the transversal part of the Kähler form is closed (for a proof of this result, see e.g. $[15$, Theorem 3.1]). We recall that a form is called horizontal with respect to a foliation $\mathcal{F}$ if its interior product with any vector field tangent to the foliation vanishes and is called basic if in addition its Lie derivative along a vector field tangent to the foliation also vanishes. In the sequel we shall use the basic versions of the standard operators acting on $\Omega_{B}^{*}(M)$, the space of basic forms: 
$\Delta_{B}$ is the basic Laplace operator, $L_{B}$ is the exterior multiplication with the transversal Kähler form and $\Lambda_{B}$ its adjoint with respect to the transversal metric. For details on these operators and their properties we refer the reader to [14, Chapter 12]. Our main result, which is an analogue of Theorem 2.10 for Sasakian structures, puts severe restrictions on formal Vaisman metrics:

Theorem 2.12 ([11]). Let $\left(M^{2 m}, g, J\right)$ be a compact Vaisman manifold. The metric $g$ is formal if and only if $b_{p}(M)=0$ for $2 \leq p \leq 2 m-2$ and $b_{1}(M)=$ $b_{2 m-1}(M)=1$, i.e. $M$ is a cohomological Hopf manifold.

Proof: Let $\gamma \in \Omega^{p}(M)$ be a harmonic form on $M$ for some $p, 1 \leq p \leq m-1$. By [15, Theorem 4.1], $\gamma$ has the following form:

$$
\gamma=\alpha+\theta \wedge \beta
$$

with $\alpha$ and $\beta$ basic, transversally harmonic and transversally primitive.

Since $\alpha$ is basic, $J \alpha$ is also a basic $p$-form that is transversally harmonic and transversally primitive:

$$
\Delta_{B}(J \alpha)=0, \quad \Lambda_{B}(J \alpha)=0,
$$

because $\Delta_{B}$ and $\Lambda_{B}$ both commute with the transversal complex structure $J$ (as the foliation is transversally Kähler). Again from [15, Theorem 4.1], by taking $\beta=0$, it follows that $J \alpha$ is a harmonic form on $M: \Delta(J \alpha)=0$.

The assumption that $g$ is formal implies that $\alpha \wedge J \alpha$ is harmonic on $M$ and in particular coclosed: $\delta(\alpha \wedge J \alpha)=0$. According to [15], this implies that $\alpha \wedge J \alpha$ is transversally primitive: $\Lambda_{B}(\alpha \wedge J \alpha)=0$.

On the other hand, it is proven in [5, Proposition 2.2] that for primitive forms $\eta, \mu \in \Lambda^{p} V$, where $(V, g, J)$ is any Hermitian vector space, the following algebraic relation holds:

$$
(\Lambda)^{p}(\eta \wedge \mu)=(-1)^{\frac{p(p-1)}{2}} p !\langle\eta, J \mu\rangle,
$$

where $J$ is the extension of the complex structure to $\Lambda^{*} V$ defined by:

$$
(J \eta)\left(v_{1}, \ldots, v_{p}\right):=\eta\left(J v_{1}, \ldots, J v_{p}\right), \quad \text { for all } \eta \in \Lambda^{p} V, v_{1}, \ldots, v_{p} \in V .
$$

We apply the above formula to the transversal Kähler geometry and obtain that $\alpha$ vanishes everywhere:

$$
0=\left(\Lambda_{B}\right)^{p}(\alpha \wedge J \alpha)=(-1)^{\frac{p(p+1)}{2}} p !\langle\alpha, \alpha\rangle .
$$

The same argument as above applied to $\beta \in \Omega_{B}^{p-1}(M)$ shows that $\beta$ is identically zero if $p \geq 2$. Thus, $\gamma=0$ for $2 \leq p \leq m-1$, which proves that:

$$
b_{2}(M)=\cdots=b_{m-1}(M)=0 .
$$


If $p=1$, then $\beta$ is a basic function, which is transversally harmonic, so that $\beta$ is a constant. Thus $\gamma$ is a multiple of $\theta$, showing that the space of harmonic 1-forms on $M$ is 1-dimensional: $b_{1}(M)=1$.

It remains to show that the Betti number in the middle dimension, $b_{m}(M)$, also vanishes. This follows from Proposition 2.5 applied to $p=1, k=m-1$ and $l=2$.

The converse is clear, since the space of harmonic forms with respect to the Vaisman metric $g$ is spanned by $\left\{1, \theta, * \theta, d v^{\circ} l_{g}\right\}$ and thus the only product of harmonic forms which is not trivial is $\theta \wedge * \theta=g(\theta, \theta) d v o l_{g}$, which is harmonic because $\theta$ has constant length, being a parallel 1-form.

\section{References}

[1] F.A. Belgun, On the metric structure of non-Kähler complex surfaces, Math. Ann. 317 (2000), 1-40.

[2] V. Bangert, M. Katz, An optimal Loewner-type systolic inequality and harmonic one-forms of constant norm, Comm. Anal. Geom. 12 (2004) no.3, 703-732.

[3] P. Deligne, Ph. Griffith, J. Morgan, D. Sullivan, Real homotopy theory of Kähler manifolds, Inventiones Mathematicae 29 (1975) no.3, 245-274.

[4] S. Dragomir and L. Ornea, Locally conformal Kähler geometry, Progress in Math. 155, Birkhäuser, Boston, Basel, 1998.

[5] J.-F. Grosjean, P.-A. Nagy, On the cohomology algebra of some classes of geometrically formal manifolds, Proc. Lond. Math. Soc. 98 (2009), 607-630.

[6] D. Kotschick, On products of harmonic forms, Duke Math. J. 107, (2001), $521-531$.

[7] D. Kotschick, S. Terzić, On formality of generalized symmetric spaces, Math. Proc. Cambridge Philos. Soc. 134 (2003), 491-505.

[8] D. Kotschick, S. Terzić, Geometric formality of homogeneous spaces and of biquotients, Pacific J. Math. 249 (2011), 157-176.

[9] P.-A. Nagy, On length and product of harmonic forms in Kähler geometry, Math. Z. 254, 199-218.

[10] P.-A. Nagy, C. Vernicos, The length of harmonic forms on a compact Riemannian manifold, Trans. Amer. Math. Soc. 356 (2004), 2501-2513. 
[11] L. Ornea, M. Pilca, Remarks on the product of harmonic forms, Pacific J. Math. 250 (2011), $353-363$.

[12] L. Ornea, M. Verbitsky, Structure theorem for compact Vaisman manifolds, Math. Res. Lett. 10 (2003), 799-805.

[13] D. Sullivan, Infinitesimal computations in topology, Inst. Hautes Études Sci. Publ. Math. 47 (1977), 269-331.

[14] Ph. Tondeur, Foliations on Riemannian Manifolds, Springer-Verlag, New York, 1988.

[15] I. Vaisman, Generalized Hopf manifolds, Geom. Dedicata 13 (1982), 231255 .

Mihaela PILCA,

Fakultät für Mathematik,

Universität Regensburg,

Universitätsstr. 31, D-93053 Regensburg, Germany.

Email: Mihaela.Pilca@mathematik.uni-regensburg.de

Mihaela PILCA,

Institute of Mathematics "Simion Stoilow" of the Romanian Academy,

21, Calea Grivitei str. 010702-Bucharest, Romania. 